\title{
Expression and significance of autophagy genes LC3, Beclin1 and MMP-2 in endometriosis
}

\author{
XIAOYU SUI ${ }^{1-3}$, YADONG LI ${ }^{4}$, YURONG SUN ${ }^{5}$, CHUNYAN LI $^{5}$, XIULAN LI $^{1}$ and GUIYU ZHANG ${ }^{2,3}$ \\ ${ }^{1}$ Department of Obstetrics and Gynecology, The First Affiliated Hospital of Qiqihar Medical University, \\ Qiqihar, Heilongjiang 161041; ${ }^{2}$ Department of Obstetrics and Gynecology, Qilu Hospital of Shandong University; \\ ${ }^{3}$ Cheeloo College of Medicine, Shandong University, Jinan, Shandong 250012; ${ }^{4}$ Clinical Laboratory, \\ The Third Affiliated Hospital of Qiqihar Medical University, Qiqihar, Heilongjiang 161006; \\ ${ }^{5}$ Clinicopathological Diagnosis Center, Qiqihar Medical University, \\ Qiqihar, Heilongjiang 161000, P.R. China
}

Received March 27, 2018; Accepted June 15, 2018

DOI: $10.3892 /$ etm.2018.6362

\begin{abstract}
Expression of autophagy-related proteins, microtubule-associated protein light chain 3 (LC3) and Beclin1, and matrix metalloproteinase-2 (MMP-2) was investigated in serum and peritoneal fluid of patients with endometriosis (EM). The messenger ribonucleic acid (mRNA) expression levels of MMP-2,LC3 and Beclin1 in endometrial tissues of EM patients and correlation of these genes with EM and their significance were evaluated. The serum, peritoneal fluid and endometrial tissues of 84 patients treated in The First Affiliated Hospital of Qiqihar Medical University (Qiqihar, China) from March 2016 to March 2017 were collected. The serum, peritoneal fluid and endometrial tissues of $42 \mathrm{EM}$ patients were used as the experimental group, while those of 42 non-EM patients were used as the control group. The levels of LC3, Beclin1 and MMP-2 in serum and peritoneal fluid of EM patients and non-EM patients were quantitatively detected via enzyme-linked immunosorbent assay (ELISA), followed by comparative analysis based on data in both groups. In addition, mRNA expression of LC3, Beclin1 and MMP-2 in the endometrium in both groups were detected via reverse transcription-quantitative polymerase chain reaction (RT-qPCR), and differences in expression of these genes between the groups were analyzed and evaluated. Correlation of LC3, Beclin1 and MMP-2 with EM was explored. Results of ELISA showed that levels of LC3 and Beclin1 in the EM group were significantly lower than those in the control group, while levels of MMP-2 in serum and peritoneal fluid of the EM group were significantly higher than those in the control group $(\mathrm{P}<0.05)$. Results of RT-qPCR revealed that mRNA expression of LC3 and Beclin1 in the
\end{abstract}

Correspondence to: Dr Guiyu Zhang, Department of Obstetrics and Gynecology, Qilu Hospital of Shandong University, 107 Wenhua West Road, Jinan, Shandong 250012, P.R. China

E-mail: ghy5nc@163.com; qlzgy55566@163.com

Key words: endometriosis, autophagy, LC3, Beclin1, MMP-2 endometrium of patients in the EM group were obviously decreased compared with those in the control group, while the expression of MMP-2 was high, and differences in expression were statistically significant $(\mathrm{P}<0.05)$. The expression of MMP-2 is high, and expression of LC3 and Beclin1 is low in serum, peritoneal fluid and endometrium of EM patients, and investigating the expression of MMP-2, LC3 and Beclin1 in $\mathrm{EM}$ is helpful to further clarify the pathogenesis of EM, and guide the clinical diagnosis and treatment.

\section{Introduction}

Endometriosis (EM) refers to a common and frequently-occurring disease in women of childbearing age due to the implantation and growth of active endometrial cells outside the endometrium, and the occurrence has a certain correlation with levels of hormones in the body (1). In recent years, the incidence rate of EM has been as high as 10-15\%, showing an increasing trend (2). Although EM is a kind of benign tumor, its invasive feature and capacity are similar to those of malignant tumors, so it is difficult to cure. The most prominent clinical manifestations of EM are dysmenorrhea, irregular menstruation, infertility and pelvic pain, which seriously affect the physical and mental health of patients, and bring immense suffering to patients and their families (3). Under normal circumstances, endometrial cells grow in the uterine cavity, but the uterine cavity can be connected to the pelvic cavity through the fallopian tube, so ectopic growth will occur in endometrial cells when they enter the pelvic cavity through the fallopian tube, resulting in EM (4). The pathogenesis of EM mainly includes blood reflux, endometrial implantation, abnormal immune response in the body, theory of heredity, theory of lymphatic and venous dissemination, iatrogenic endometrial transplantation, metaplasia and benign metastasis. However, its mechanism of occurrence remains unclear so basic research on EM is a widespread concern for experts and scholars worldwide (5). At present, the clinical treatment of EM is dominated by surgery and endocrine therapy, but the effect is unsatisfactory, and it is difficult to eradicate the focus with a high recurrence rate. In recent years, with 
the increasing deepening of immunology research, the roles of cytokines in the pathogenesis of EM have received more attention (6). Matrix metalloproteinase-2 (MMP-2) is involved in a variety of pathophysiological processes mainly through regulating the homeostasis of extracellular matrix and regulating the synthesis and degradation processes of extracellular matrix protein, which is possibly related to the occurrence of EM (7). Microtubule-associated protein light chain 3 (LC3) and Beclin1 are specific marker proteins of autophagy, as well as important regulators of autophagy. In recent years, autophagy and various diseases have been widely studied, but there are no reports on the correlation of autophagy-related genes (LC3 and Beclin1) with EM at present (8). In this study, the expression and clinical significance of LC3, Beclin1 and MMP-2 in serum, peritoneal fluid and endometrial tissues of patients with EM were investigated, so as to provide new targets for the clinical diagnosis and treatment of EM.

\section{Materials and methods}

General data. EM patients and non-EM patients treated in The First Affiliated Hospital of Qiqihar Medical University (Qiqihar, China) from March 2016 to March 2017 were selected. In EM group, there were 42 patients, aged 21-42 years, with an average of $29.5 \pm 6.0$ years. In non-EM group, there were 42 patients, aged 18-40 years, with an average of $31.5 \pm 5.0$ years. There was no statistically significant difference in the age between the two groups $(\mathrm{P}>0.05)$. The mean menstrual cycle of patients in both groups was $28.59 \pm 3.27$ days, and the mean menstrual period was $6.45 \pm 0.50$ days. Inclusion criteria were: patients with normal results of each routine test during the collection of specimens, patients diagnosed with EM or non-EM via pathological examination, patients without adenomyosis, polycystic ovary syndrome, gynecological endocrine diseases, immune system diseases, tumors, pelvic inflammation and other major medical and surgical diseases, patients receiving no hormonal therapy within 3 months, and patients without intrauterine device. This study was approved by the Ethics Committee of The First Affiliated Hospital of Qiqihar Medical University (Qiqihar, China), and patients and their families had been informed of the purpose, method and significance of this study. All patients volunteered to participate in the project and signed the informed consent.

\section{Methods}

Specimen collection. After $5 \mathrm{ml}$ fasting peripheral venous blood was drawn from EM and non-EM patients in the early morning on the second day after admission, it was centrifuged at $10,500 \mathrm{x} \mathrm{g}$ for $10 \mathrm{~min}$, and the supernatant was transferred into an autoclaved $1.5 \mathrm{ml}$ Eppendorf (EP) tube by using a pipette and stored in a refrigerator at $-20^{\circ} \mathrm{C}$. The peritoneal fluid not contaminated by the blood was sucked from EM and non-EM patients under the laparoscope during surgery, and centrifuged at $2,750 \mathrm{x}$ g for $10 \mathrm{~min}$, and then the supernatant was taken and stored in the refrigerator at $-20^{\circ} \mathrm{C}$. To collect endometrial tissues, patients received surgery at 3-7 days after menstruation, before which a small amount of endometrium in the proliferative stage was sucked by using a disposable endometrial aspirator. The fresh endometrial tissues were treated with RNAlater, rapidly frozen in liquid nitrogen and stored in a refrigerator at $-80^{\circ} \mathrm{C}$.
Enzyme-linked immunosorbent assay (ELISA). The concentrations of LC3, Beclin1 and MMP-2 were determined via ELISA, and all procedures were performed in strict accordance with the instructions of the kit. First, the serum in each group was extracted and centrifuged at $10,500 \mathrm{xg}$ and $4^{\circ} \mathrm{C}$ for $10 \mathrm{~min}$. The supernatant of the peritoneal fluid was extracted and centrifuged at $2,750 \mathrm{x} \mathrm{g}$ and $4^{\circ} \mathrm{C}$ for $10 \mathrm{~min}$. Then the supernatant was taken and transferred to a $1.5 \mathrm{ml}$ centrifuge tube to detect the concentrations of MMP-2, LC3 and Beclin1, respectively, according to instructions of the MMP-2, LC3 and Beclin1 (total) ELISA kits. Finally, the optical density (OD) value was read at a wavelength of $450 \mathrm{~nm}$ by using a microplate reader (Bio-Rad, Hercules, CA, USA), and the zero well was set as the control well, and the standard curve was drawn with the OD value as vertical coordinate and the concentration as horizontal coordinate. Based on the OD value of specimen, the corresponding concentration was found on the coordinate. If the specimen had been diluted, the final result was multiplied by the dilution factor. The standard substance was used for the detection of repeated well, and the average was taken to calculate the coefficient of linear correlation equation, so as to establish the standard equation. The repeated well was set for each specimen, and the average was taken, recorded and substituted into the above regression equation to calculate the concentration of each specimen.

Instruments and reagents. ELISA kit (Hangzhou MultiSciences Biotech Co., Ltd., Hangzhou, China), Nanodrop and microplate reader (both from Thermo Fisher Scientific, Inc., Waltham, MA, USA), high-speed low-temperature centrifugation (Hunan Kaida Scientific Instrument Co., Ltd., Changsha, China), 1,000 $\mu 1,200 \mu \mathrm{l}, 20 \mu \mathrm{l}$ and $2 \mu \mathrm{l}$ pipettes (Thermo Fisher Scientific, Inc.), $1.5 \mathrm{ml}$ EP tube, 1,000 $\mu \mathrm{l}, 200 \mu \mathrm{l}, 20 \mu \mathrm{l}$ and $2 \mu \mathrm{l}$ pipette tips (Shenzhen Baluoke Technology Co., Ltd., Shenzhen, China), reverse transcription-quantitative polymerase chain reaction (RT-qPCR) instrument, and reverse transcription machine (Roche Applied Science, Penzberg, Germany), reverse transcription kit (ABI; Thermo Fisher Scientific, Inc.), ice plate, RNAlater, and SYBR (Vazyme Biotech Co., Ltd, Nanjing, China), isopropanol, chloroform, and absolute ethyl alcohol (all from Zhengzhou Yibang Industrial Co., Ltd., Zhengzhou, China), and diethylpyrocarbonate (DEPC)-treated water (Biosharp Life Sciences, Hefei, China).

$R T-q P C R$. The messenger ribonucleic acid (mRNA) expression levels of LC3, Beclin1 and MMP-2 in endometrial tissues of EM patients and non-EM patients were detected, and fresh endometrial tissues were treated with RNAlater to prevent RNA degradation. Endometrial tissues were ground to powder by using a grinding rod, and a small number of endometrial tissues were lysed with TRIzol reagent, and the total RNA was extracted. Then the OD values were read at $260 \mathrm{~nm}$ and $280 \mathrm{~nm}$ by using Nanodrop via ultraviolet spectrophotometry (Hitachi, Tokyo, Japan), and the purity and concentration of RNA were recorded. The RT reaction system was prepared according to the instructions: a total volume of $10 \mu \mathrm{l}$, including $0.4 \mu \mathrm{l}$ deoxy-ribonucleoside triphosphate (dNTP) mixture, $0.5 \mu 1$ RNase inhibitor, 10x1 $\mu \mathrm{l}$ RT buffer, $1 \mu \mathrm{l}$ random primer, the total volume of distilled water and RNA of $2.9 \mu 1$, and the total RNA mass of $500 \mathrm{ng}$. The mixtures were mixed on an ice box, followed by RT on an RT instrument, after which RT-qPCR was performed. PCR primers were synthesized by Genechem Co., Ltd. (Shanghai, China). Amplification conditions are as 
Table I. Primer sequences in RT-qPCR.

\begin{tabular}{lll}
\hline Genes & \multicolumn{1}{c}{ Forward } & \multicolumn{1}{c}{ Reverse } \\
\hline LC3 & AAGGCGCTTACAGCTCAATG & CTGGGAGGCATAGACCATGT \\
Beclin1 & AAACGCATTTGCCATCACA & GGACCTTCAGCAGTTTACAGTCAG \\
MMP-2 & CTTCCAAGTCTGGAGCGATGT & TACCGTCAAAGGGGTATCCAT \\
GAP & CATCACTGCCACCCAGAAGAC & CCAGTGAGCTTCCCGTTCAG \\
\hline
\end{tabular}

LC3, light chain 3; MMP-2, matrix metalloproteinase-2.

follows: pre-denaturation at $94^{\circ} \mathrm{C}$ for $5 \mathrm{~min}$, denaturation at $94^{\circ} \mathrm{C}$ for $30 \mathrm{sec}$, annealing at $56^{\circ} \mathrm{C}$ for $30 \mathrm{sec}$, extension at $72^{\circ} \mathrm{C}$ for $30 \mathrm{sec}$, a total of 40 cycles of denaturation, annealing and extension, extension again at $72^{\circ} \mathrm{C}$ for $7 \mathrm{~min}$ before the end, and storage at $4^{\circ} \mathrm{C}$. The mRNA expression levels of LC3, Beclin1 and MMP-2 were calculated and quantitatively analyzed by using $\Delta \Delta \mathrm{Cq}$ with GAP as the internal control gene (Table I) (9).

Statistical analysis. Statistical Product and Service Solutions (SPSS) 17.0 statistical software (SPSS Inc., Chicago, IL, USA) was used for data analysis. Measurement data in line with the normal distribution were presented as mean \pm standard deviation, and t-test was used for the intergroup comparison. Ranked data are presented as rate, adopting the rank sum test. $\mathrm{P}<0.05$ was considered to indicate a statistically significant difference.

\section{Results}

Expression levels and comparison of serum LC3, Beclin1 and MMP-2 between EM and non-EM patients. Compared with those in the non-EM group, the expression of serum MMP-2 was significantly increased in the EM group, while the expression of autophagy-related genes (LC3 and Beclin1) was significantly decreased, and the differences were statistically significant $(\mathrm{P}<0.05)$ (Table II).

Expression levels and comparison of LC3, Beclinl and MMP-2 in peritoneal fluid between EM and non-EM patients. Compared with those in the non-EM group, the expression of MMP-2 in peritoneal fluid was significantly increased in the EM group, while the expression of autophagy-related genes (LC3 and Beclin1) were significantly decreased, and the differences were statistically significant $(\mathrm{P}<0.05)$ (Table III).

Expression levels and comparison of LC3, Beclinl and MMP-2 in endometrial tissues between EM and non-EM patients. Compared with those in the non-EM group, the mRNA expression of MMP-2 in endometrial tissues was remarkably increased in the EM group, while the mRNA expression of autophagy-related genes (LC3 and Beclin1) was remarkably decreased, and the differences were statistically significant $(\mathrm{P}<0.05)$ (Fig. 1).

\section{Discussion}

EM is a common gynecological disease caused by the location of endometrium outside the uterus, and patients mainly have
Table II. Comparison of expression levels of serum LC3, Beclin1 and MMP-2 between the two groups of patients.

\begin{tabular}{lcccl}
\hline Groups & $\mathrm{n}$ & MMP-2 $(\mu \mathrm{g} / \mathrm{l})$ & $\mathrm{LC} 3(\mu \mathrm{g} / \mathrm{l})$ & Beclin1 $(\mu \mathrm{g} / \mathrm{l})$ \\
\hline EM & 42 & $226.1 \pm 49.0$ & $36.86 \pm 16.86$ & $25.16 \pm 9.89$ \\
Non-EM & 42 & $130.5 \pm 13.5$ & $75.54 \pm 16.09$ & $55.45 \pm 17.14$ \\
\hline
\end{tabular}

LC3, light chain 3; MMP-2, matrix metalloproteinase-2; EM, endometriosis.

Table III. Comparison of expression levels of LC3, Beclin1 and MMP-2 in peritoneal fluid between the two groups of patients.

\begin{tabular}{lcccc}
\hline Groups & $\mathrm{n}$ & MMP-2 $(\mu \mathrm{g} / \mathrm{l})$ & $\mathrm{LC} 3(\mu \mathrm{g} / \mathrm{l})$ & Beclin1 $(\mu \mathrm{g} / \mathrm{l})$ \\
\hline EM & 42 & $48.3 \pm 8.4$ & $17.64 \pm 10.21$ & $10.17 \pm 4.14$ \\
Non-EM & 42 & $22.5 \pm 5.2$ & $38.97 \pm 11.85$ & $20.53 \pm 5.51$ \\
\hline
\end{tabular}

LC3, light chain 3; MMP-2, matrix metalloproteinase-2; EM, endometriosis.

clinical symptoms such as dysmenorrhea, chronic pelvic pain, dyspareunia, acute abdominal pain, abnormal menstruation or infertility (10). EM seriously affects the reproductive health and quality of life of patients. Therefore, it is an issue to be solved in the field of gynecology and basic science to study EM deeply and find out more specific and effective methods for diagnosis, treatment and prevention (11). Although EM is a kind of benign tumor, it has attracted the attention of experts and scholars worldwide due to its biological characteristics, such as metastasis, development, implantation, erosion, tissue destruction and potential malignant transformation (12). Moreover, its pathogenesis is complex and not fully understood, which is currently believed to be related to a variety of bioactive factors, such as genetic material, steroid hormones, environmental factors and immune molecules $(13,14)$.

In autophagy, eukaryotic cells provide cells with the required amino acids and fatty acids under the state of stress to maintain the cellular environmental homeostasis when eliminating and degrading their damaged organelles by using lysosomes. Autophagy is related to a variety of autophagyrelated genes, among which LC3 and Beclin1 are important autophagic factors (15). LC3, as a homologous gene with 

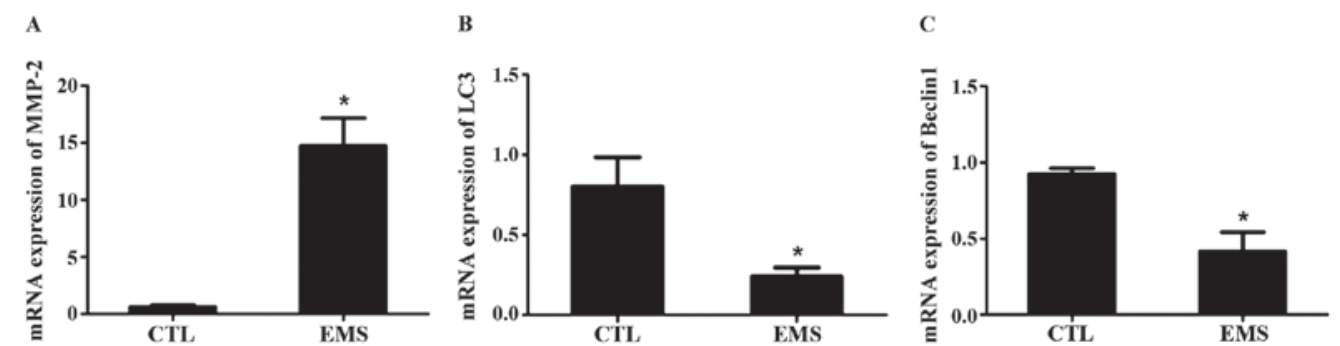

Figure 1. mRNA expression levels of LC3, Beclin1 and MMP-2 in endometrial tissues of non-EM and EM patients. Compared with those in the non-EM group, (A) the mRNA expression of MMP-2 in EM patients is remarkably increased, (B) while the mRNA expression of LC3 and (C) Beclin1 is remarkably decreased. LC3, light chain 3; MMP-2, matrix metalloproteinase-2; EM, endometriosis. ${ }^{*} \mathrm{P}<0.05$.

autophagy-related gene Atg8 in yeast, and a specific marker of autophagosome, which expression level can directly reflect the level of autophagy (16). Beclin1, also known as BECN 1, is a specific gene of autophagy and an autophagy-related tumor suppressor gene, as well as a direct regulator of autophagy in mammals (17). In recent years, research on correlation of autophagy and tumor has become a hot spot, and studies have shown that the autophagy capacity varies in a variety of tumors. EM possesses many biological characteristics of tumor diseases, such as metastasis, implantation and recurrence $(18,19)$. It has been reported that autophagy is related to various malignant tumors. For example, the decline in autophagy activity is associated with the occurrence and development of pancreatic cancer, breast cancer, cervical squamous cell carcinoma, colon cancer and ovarian cancer, demonstrating that abnormal expression of autophagy-related genes have a close correlation with the occurrence of tumor (20-24). At present, there are few reports on the correlation of occurrence of EM with LC3 and Beclin1. This study mainly focused on the correlations of LC3 and Beclin1 with EM and their clinical significance.

MMP-2 is a kind of collagenase, which can exert its enzymolysis under the activation of various activation factors. Its enzymatic substrates are mainly type IV, V, VII, IX and $\mathrm{X}$ collagen, fibronectin and elastin. Therefore, MMP-2 plays a certain role in the process of tumor cell shedding, invasion and migration (25). According to the data displayed, the high expression of MMP-2 can enable it to exert strong proteolysis, degrade the basement membrane components around ectopic endometrial tissues, and affect the correlation among mesenchymal cells, thus invading the peritoneum. At the same time, MMP-2 can promote intercellular adhesion and angiogenesis, and accelerate the growth of ectopic endometrial tissues, thereby expanding the focus and aggravating the disease of patients (26). In addition, MMP-2 can regulate the updating of extracellular matrix, while the synthesis and degradation processes of extracellular matrix depend on MMP-2. Once such regulation becomes imbalanced, many diseases and pathological changes will be caused. This phenomenon plays an extremely important role in the occurrence and development of EM, and MMP-2 has an important relation with EM, providing a new idea and direction for the treatment of EM (27).

In this experiment, the serum, peritoneal fluid and endometrial tissues were collected from 84 patients, including 42 EM patients and 42 non-EM patients. The expression levels of MMP-2, LC3 and Beclin1 in serum and peritoneal fluid of non-EM and EM patients were detected, respectively. Results manifested that the expression of MMP-2 in serum and peritoneal fluid of EM patients was significantly increased compared with those of non-EM patients, while expression of autophagyrelated genes (LC3 and Beclin1) was decreased significantly. In addition, the mRNA levels of MMP-2, LC3 and Beclin1 in endometrial tissues of non-EM and EM patients were detected via qRT-PC. Results revealed that compared with those in the control group, the mRNA level of MMP-2 showed an increasing trend in endometrial tissues of EM patients, while mRNA levels of LC3 and Beclin1 showed decreasing trends in EM patients, and differences were statistically significant. The above results indicate that LC3 and Beclin1 are expressed at low level and MMP-2 is highly expressed in EM patients. These three genes can be used as diagnostic indexes and biomarkers for EM patients, providing a simpler and faster method for clinical diagnosis of EM.

This study detected the expression of MMP-2, LC3 and Beclin1 in serum, peritoneal fluid and endometrium of EM and non-EM patients via RT-qPCR and ELISA, analyzed the roles and clinical significance of MMP-2, LC3 and Beclin1 in the occurrence and development of EM, and explored the correlation of the three genes with the occurrence of EM, so as to provide an important theoretical basis for the pathogenesis of EM, and provide new ideas and effective markers for the clinical treatment of EM.

\section{Acknowledgements}

Not applicable.

\section{Funding}

This study was supported by the Qiqihar Science and Technology Project (SFGG-201714).

\section{Availability of data and materials}

The datasets used and/or analyzed during the present study are available from the corresponding author on reasonable request.

\section{Authors' contributions}

XS was involved in the conception and design of the study. GZ collected the specimens. YL and CL performed ELISA. YS and XL were responsible for PCR. All the authors have read and approved the final study. 


\section{Ethics approval and consent to participate}

The study was approved by the Ethics Committee of The First Affiliated Hospital of Qiqihar Medical University (Qiqihar, China). Patients who participated in this study, signed an informed consent and had complete clinical data. Signed informed consents were obtained from the patients or guardians.

\section{Patient consent for publication}

Not applicable.

\section{Competing interests}

The authors declare that they have no competing interests.

\section{References}

1. Hudelist G, Tammaa A, Aas-Eng MK, Kirchner L, Fritzer N, Nemeth Z and Lamche M: Outcome of sonography-based minimally invasive surgery for deep infiltrating endometriosis of the ureter and urinary bladder - a retrospective cohort study. Acta Obstet Gynecol Scand 97: 277-284, 2018.

2. Raad J, Sonigo C, Tran C, Sifer C, Durnerin IC and Grynberg M: Oocyte vitrification for preserving fertility in patients with endometriosis: First observational cohort study... and many unresolved questions. Letter to the Editor. Eur J Obstet Gynecol Reprod Biol 220: 140-141, 2018.

3. Yang HL, Mei J, Chang KK, Zhou WJ, Huang LQ and Li MQ: Autophagy in endometriosis. Am J Transl Res 9: 4707-4725, 2017.

4. Orazov MR, Radzinskiy VY, Khamoshina MB, Nosenko EN, Tokaeva ES, Barsegyan LK and Zakirova YR: Histamine metabolism disorder in pathogenesis of chronic pelvic pain in patients with external genital endometriosis. Patol Fiziol Eksp Ter 61: 56-60, 2017 (In Russian).

5. Shukla PS and Schwartz C: Extrauterine, extragenital endometrial stromal sarcoma in the absence of endometriosis: A rare entity. Int J Gynecol Pathol: Nov 28, 2017 (Epub ahead of print).

6. Nada AM, El-Noury A, Al-Inany H, Bibars M, Taha T, Salama S, Hassan F and Zein E: Effect of laser-assisted zona thinning, during assisted reproduction, on pregnancy outcome in women with endometriosis: Randomized controlled trial. Arch Gynecol Obstet 297: 521-528, 2018.

7. Mohagheghi S, Khodadadi I, Karami M, Amiri I and Tavilani H: Gene polymorphism of matrix metalloproteinase 9 in asthenozoospermic male subjects. Int J Fertil Steril 11: 247-252, 2018.

8. Wu RF, Huang ZX, Ran J, Dai SJ, Lin DC, Ng TW, Chen QX and Chen QH: Lipoxin A4 suppresses estrogen-induced epithelial-mesenchymal transition via ALXR-dependent manner in endometriosis. Reprod Sci 25: 566-578, 2018.

9. Livak KJ and Schmittgen TD: Analysis of relative geneexpression data using real-time quantitative PCR and the 2(-Delta Delta C(T)) Method. METHODS 25: 402-408, 2001.

10. Park S, Lim W, Bazer FW and Song G: Apigenin induces ROS-dependent apoptosis and ER stress in human endometriosis cells. J Cell Physiol 233: 3055-3065, 2018.

11. Jana S, Chatterjee K, Ray AK, DasMahapatra P and Swarnakar S: Regulation of matrix metalloproteinase-2 activity by COX-2-PGE2-pAKT axis promotes angiogenesis in endometriosis. PLoS One 11: e0163540, 2016.

12. Yang WW, Hong L, Xu XX, Wang Q, Huang JL and Jiang L: Regulation of miR-33b on endometriosis and expression of related factors. Eur Rev Med Pharmacol Sci 21: 2027-2033, 2017.
13. Chen YS, Langhammer T, Westhofen $M$ and Lorenzen $\mathrm{J}$ : Relationship between matrix metalloproteinases MMP-2, MMP-9, tissue inhibitor of matrix metalloproteinases-1 and IL-5, IL-8 in nasal polyps. Allergy 62: 66-72, 2007.

14. Yang H, Liu J, Fan Y, Guo Q, Ge L, Yu N, Zheng X, Dou Y and Zheng S: Associations between various possible promoter polymorphisms of MMPs genes and endometriosis risk: A meta-analysis. Eur J Obstet Gynecol Reprod Biol 205: 174-188, 2016.

15. Katagiri H, Nakayama K, Razia S, Nakamura K, Sato E, Ishibashi T, Ishikawa M, Iida K, Ishikawa N, Otsuki Y, et al: Loss of autophagy-related protein Beclin 1 may define poor prognosis in ovarian clear cell carcinomas. Int J Oncol 47: 2037-2044, 2015.

16. Shi J, Shi S, Wu B, Zhang J, Li Y, Wu X, Zhang J, Wang K, Zhao B, Cai W, et al: Autophagy protein LC3 regulates the fibrosis of hypertrophic scar by controlling Bcl-xL in dermal fibroblasts. Oncotarget 8: 93757-93770, 2017.

17. Zhu ML, Lu JX, Pan GP, Ping S, Zhao FR, Qi HT, Yu HY, Jian X, Wan GR and Li P: Traditional Chinese medicine Ka-Sai-Ping suppresses the growths of gastric cancers via induction of autophagy. Oncotarget 8: 95075-95082, 2017.

18. Machado DE, Rodrigues-Baptista KC, Alessandra-Perini J, Soares de Moura R, Santos TA, Pereira KG, Marinho da Silva Y, Souza PJ, Nasciutti LE and Perini JA: Euterpe oleracea extract (Açaí) is a promising novel pharmacological therapeutic treatment for experimental endometriosis. PLoS One 11: e0166059, 2016.

19. Ye H, He Y, Wang J, Song T, Lan Z, Zhao Y and Xi M: Effect of matrix metalloproteinase promoter polymorphisms on endometriosis and adenomyosis risk: Evidence from a meta-analysis. J Genet 95: 611-619, 2016.

20. Boone BA, Zeh RH and Bahary N: Autophagy inhibition in pancreatic adenocarcinoma. Clin Colorectal Cancer 17: 25-31, 2017.

21. Lin SC, Chu PY, Liao WT, Wu MY, Tsui KH, Lin LT, Huang $\mathrm{CH}$, Chen LL and Li CJ: Glycyrrhizic acid induces human MDA-MB-231 breast cancer cell death and autophagy via the ROS-mitochondrial pathway. Oncol Rep 39: 703-710, 2018.

22. Fan T, Pi H, Li M, Ren Z, He Z, Zhu F, Tian L, Tu M, Xie J, Liu M, et al: Inhibiting MT2-TFE3-dependent autophagy enhances melatonin-induced apoptosis in tongue squamous cell carcinoma. J Pineal Res 64: 64, 2018.

23. Rubio MF, Lira MC, Rosa FD, Sambresqui AD, Salazar Güemes MC and Costas MA: RAC3 influences the chemoresistance of colon cancer cells through autophagy and apoptosis inhibition. Cancer Cell Int 17: 111, 2017.

24. Peng Q, Qin J, Zhang Y, Cheng X, Wang X, Lu W, Xie X and Zhang S: Autophagy maintains the stemness of ovarian cancer stem cells by FOXA2. J Exp Clin Cancer Res 36: 171, 2017.

25. Sultana S, Dey R and Bishayi B: Dual neutralization of TNFR-2 and MMP-2 regulates the severity of $\mathrm{S}$. aureus induced septic arthritis correlating alteration in the level of interferon gamma and interleukin-10 in terms of TNFR2 blocking. Immunol Res 66: 97-119, 2018.

26. Jana S, Rudra DS, Paul S and Snehasikta S: Curcumin delays endometriosis development by inhibiting MMP-2 activity. Indian J Biochem Biophys 49: 342-348, 2012.

27. Pourjafar M, Saidijam M, Etemadi K and Najafi R: All-trans retinoic acid enhances in vitro mesenchymal stem cells migration by targeting matrix metalloproteinases 2 and 9 . Biotechnol Lett 39: 1263-1268, 2017.

This work is licensed under a Creative Commons Attribution-NonCommercial-NoDerivatives 4.0 International (CC BY-NC-ND 4.0) License. 